\title{
Modelling and analysis of the global stability of Blasius boundary-layer flow interacting with a compliant wall
}

\author{
$\underline{\text { K. Tsigklifis }}^{a}$ and A.D. Lucey ${ }^{a}$ \\ ${ }^{a}$ Fluid Dynamics Research Group \\ Curtin University of Technology, Perth, WA 6845, Australia \\ Email:k.tsigklifis@curtin.edu.au
}

\begin{abstract}
Theoretical and experimental studies have shown that compliant walls are able to reduce the growth rates of unstable Tollmien-Schlichting waves (TSWs) that are the conventional route to boundarylayer transition in low-disturbance environments. Accordingly, transition can be postponed by an appropriately designed compliant coating adhered to an otherwise rigid surface, thereby leading to a potentially significant reduction of skin-friction drag in marine applications. The more compliant the wall, the greater is the suppression of TSWs. However, the compliant wall can also support unstable wall-based waves that typically occur when the wall is too compliant and thereby undermine the overall flowstabilization strategy. Accordingly, when designing useful complaint coatings, it is necessary to take into account all of the possible instabilities of the fluid-structure interaction (FSI) system.

The majority of previous studies utilize local-stability analyses based upon the assumptions of an infinitely long compliant wall and parallel-flow to identify and characterise the system instabilities while numerical simulation has been used for walls of finite extent. In contrast, we carry out a bi-global linear stability analysis in the present study of the FSI system. We model the flow using a combination of vortex and source boundary-element sheets on a computational grid while the dynamics of a plate-spring, Kramer-type, complaint wall are represented in finite-difference form. The assembled FSI system is then couched as an eigenvalue problem and the eigenvalues of the various flow- and wall-based instabilities are analyzed for a range of system parameters.

The key findings of the study are that coalescence - or resonance - of one of the structural eigenmodes with either the most unstable TSW or a travelling-wave flutter (TWF) mode can occur. This renders the convective nature of these instabilities to become global for a finite compliant wall. A local analysis of the temporally unstable modes shows that besides the TSW and TWF modes, a divergence-type mode associated with the structural behaviour can additionally yield global instability. Finally, a non-modal analysis reveals that the behaviour of flow-based instabilities over a structurally damped compliant wall in response to an initial disturbance shows slightly lower transient growth and energy advection than occurs over a rigid wall in the sub-resonance combination of wall and flow parameters. However, for system conditions that yield resonance-type behaviour, transient growth is significantly larger than that which occurs over a rigid wall.
\end{abstract}

Keywords: $\quad$ Fluid-structure interaction, Global stability, Blasius flow, Compliant wall, Non-modal analysis 


\section{INTRODUCTION}

Even though local stability studies (e.g. Carpenter \& Garrad 1985, Dixon et al. 1993) have revealed the main physics behind the phenomena associated with the interaction of boundary-layer flow and a compliant wall the fluid-structure system (FSI) depicted in Fig. 1 - there remain limitations to the analysis due to its local nature. More specifically, the end conditions of the compliant wall cannot be taken into account due to the assumed homogeneous streamwise direction. However, numerical simulations (Lucey \& Carpenter 1992) and global stability analysis in the potential-flow regime (Pitman \& Lucey 2009) have, inter alia, shown that the effect of the structural damping on divergence instability is predicted correctly only when one considers the compliant wall as a finite structure. Furthermore, it is not known whether the eigen-frequencies of the finite structure interact with the frequencies of viscous-flow instabilities and if this can manifest itself in a local stability analysis.

Preliminary work on the global stability analysis of two-dimensional (2D) plane-channel Poisseuille flow over a compliant wall with periodic boundary conditions was conducted by Pitman \& Lucey (2010) using a velocity-vorticity formulation combined with a vortex/source-sheet boundary integral method. They found that a flexible wall with structural damping could stabilise the (temporal) global system by $4 \%$ at $\mathrm{Re}=6000$ (based on channel height). The present work extends this velocity-vorticity formulation and combines it with the generalized Helmholtz decomposition (Kempka et al., 1995) to study the global stability of Blasius boundary-layer flow over a compliant wall and investigate the asymptotic behaviour and transient response of the FSI system to 2D incident disturbances. Local spatial stability anlysis is also conducted in order to validate the global analysis, but also to reveal the spatial characteristics of the predicted temporal instabilities. A key contribution of the present work is that wall's fixed-end boundary conditions are incorporated and that boundary-layer growth is modelled. All of the fluid and structural eigen-frequencies and the different instability branches are obtained through the solution of a generalized eigenvalue problem. In this way, it is possible to investigate the interaction between the different mode types and identify the conditions for temporal instability of the FSI system.

\section{PROBLEM FORMULATION}

\subsection{Mean flow field}

The mean undisturbed flow of interest is the zero-pressure-gradient semi-infinite-plate Blasius boundary layer as depicted in Fig. 1.

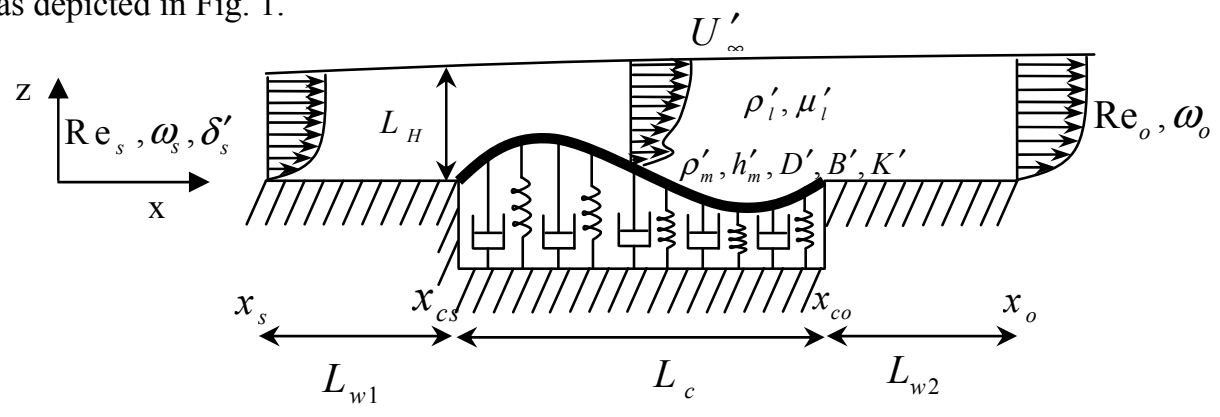

Figure 1. Schematic diagram of the studied FSI system.

The displacement thickness $\delta_{s}^{\prime}$ at the entrance $x_{s}^{\prime}$ of the flow field is assigned as the characteristic length scale, while the undisturbed-flow velocity, $U_{\infty}^{\prime}$, gives the characteristic speed, with the prime denoting a dimensional quantity. The local Reynolds number, $\mathrm{Re}$, at a position $x$ of the flow field is related to the $\mathrm{Re}_{s}$ at the entrance of the domain by $\mathrm{Re}_{x}=\gamma \sqrt{x \mathrm{Re}_{s}}$ for $\operatorname{Re}_{s}=\rho_{l}^{\prime} U_{\infty}^{\prime} \delta^{\prime} / \mu_{l}^{\prime}=\gamma \sqrt{\rho_{l}^{\prime} U_{\infty}^{\prime} x^{\prime} / \mu_{l}^{\prime}}, \quad \gamma=1.7208$. The undisturbed velocities are given by $U_{x}=d f / d H$ and $U_{z}=\gamma /\left(2 \sqrt{x \mathrm{Re}_{s}}\right)(H \cdot d f / d H-f)$ where $f(H)$ satisfies the Blasius equation,

$2 \frac{d^{3} f}{d H^{3}}+\gamma^{2} f \frac{d^{2} f}{d H^{2}}=0$, 
K. Tsigklifis and A.D. Lucey, Modelling and analysis of the global stability of Blasius boundary-layer

with $H=z \sqrt{\operatorname{Re}_{s}} /(\gamma \sqrt{x})$ subject to the boundary conditions $f(0)=d f / d H(0)=0$ and $d f / d H \rightarrow 0$, as $H \rightarrow \infty$

\subsection{Disturbance fields}

\section{Flow field}

Starting from the general velocity-vorticity disturbance formulation (Davies \& Carpenter 2001), allowing 2D disturbances and keeping only the linear velocity and vorticity terms, we obtain the following equations for the disturbances,

$$
\begin{aligned}
& \frac{\partial \omega_{y}}{\partial t}+\frac{\partial \Omega_{y}}{\partial x} u_{x}+\frac{\partial \Omega_{y}}{\partial z} u_{z}+U_{x} \frac{\partial \omega_{y}}{\partial x}+U_{z} \frac{\partial \omega_{y}}{\partial z}=\frac{1}{\mathrm{Re}_{x}}\left(\frac{\partial^{2} \omega_{y}}{\partial x^{2}}+\frac{\partial^{2} \omega_{y}}{\partial z^{2}}\right) \\
& \vec{\nabla}^{2} \vec{u}=-\vec{\nabla} \times\left(\omega_{y} \vec{e}_{y}\right)
\end{aligned}
$$

where variables of the mean undisturbed Blasius flow are assigned capital letters, while those of the disturbances are in lower case. In Eqns. (2) and (3), $u_{x}$ and $u_{z}$ are respectively the streamwise and normal components of the velocity disturbance, while $\Omega_{y}, \omega_{y}$ are respectively the mean-flow and the disturbance vorticity in the spanwise direction. Instead of solving the vector Poisson equation (3), we make use of the Helmholtz decomposition and express the disturbance flow field as the sum of a rotational and an irrotational velocity field, which is written for the $2 \mathrm{D}$ incompressible disturbance flow field as

$$
\vec{u}(\vec{x})=\int_{R \neq R_{b f}} \vec{\nabla} G\left(\vec{x}, \vec{x}^{\prime \prime}\right) \times \omega_{y}\left(\vec{x}^{\prime \prime}\right) \vec{e}_{y} d R\left(\vec{x}^{\prime \prime}\right)+\int_{R_{b f}} \vec{\nabla} G\left(\vec{x}, \vec{x}_{b f}^{\prime \prime}\right) \times \omega_{y}\left(\vec{x}_{b f}^{\prime \prime}\right) \vec{e}_{y} d R\left(\vec{x}_{b f}^{\prime \prime}\right)-\int_{S} \sigma\left(\vec{x}_{b s}^{\prime \prime}\right) \vec{\nabla} G\left(\vec{x}, \vec{x}_{b s}^{\prime \prime}\right) d S\left(\vec{x}_{b s}^{\prime \prime}\right)
$$

where, $G=-1 / 2 \pi \cdot \ln \left(\left|\vec{x}-\vec{x}^{\prime \prime}\right|\right)$ is the $2 \mathrm{D}$ infinite domain Green's function and $\sigma$ the strength of the source-sink sheet applied to the flow boundary. In the above integral expressions $R$, denotes integration in the fluid domain while $S$ denotes integration on the boundary surface. We have split the rotational part into a boundary flow field $R_{b f}$ and a domain flow field contribution, $R \neq R_{b f}$, in order to apply the tangential and normal boundary conditions at the boundary cells and surfaces.

\section{$\underline{\text { Structural solution }}$}

We use the one-dimensional (1D) beam equation with additional terms to account for a dashpot-type structural damping and a uniformly distributed spring foundation, i.e.,

$I C \frac{\partial^{2} \eta}{\partial t^{2}}+D C \frac{\partial \eta}{\partial t}+B C \frac{\partial^{4} \eta}{\partial x^{4}}+K C \eta=-p(x, 0, t)$

where, $I C=\rho_{m}^{\prime} h^{\prime} /\left(\rho_{l}^{\prime} \delta^{\prime}\right), \quad D C=D^{\prime} /\left(\rho_{l}^{\prime} U_{\infty}^{\prime}\right), \quad B C=B^{\prime} /\left(\rho_{l}^{\prime} U_{\infty}^{\prime 2} \delta_{s}^{\prime 3}\right), \quad K C=K^{\prime} \delta^{\prime} /\left(\rho_{l}^{\prime} U_{\infty}^{\prime 2}\right), \quad$ are the dimensionless coefficients of inertia, damping, flexural rigidity, and the spring-foundation stiffness respectively; $\eta(x, t)$ is the dimensionless plate displacement in the $z$ direction and $p(x, 0, t)$ the dimensionless pressure disturbance on the wall. Hinged boundary conditions are applied at the leading and trailing edges of the compliant wall, hence

$$
\eta\left(x_{c s}, t\right)=\eta\left(x_{c o}, t\right)=0, \quad \partial^{2} \eta / \partial x^{2}\left(x_{c s}, t\right)=\partial^{2} \eta / \partial x^{2}\left(x_{c o}, t\right)=0
$$

Boundary conditions

Following Ehrenstein \& Gallaire (2005), we make use of the Robin boundary conditions at the entrance $x_{s}$ and exit $x_{o}$ of the fluid domain, thus

$$
\partial u_{z} / \partial x=i a u_{z}, \quad \partial \omega_{y} / \partial x=i a \omega_{y}, \text { at } x=x_{s} \text { and } x=x_{o}
$$

but the complex wavenumber, $a$, is taken by the solution of the Orr-Sommerfeld equation at the entrance and at the exit of the fluid domain for cyclic frequencies, $\omega_{s}$ and $\omega_{o}=\left(\mathrm{Re}_{o} / \mathrm{Re}_{s}\right) \omega_{s}$, respectively. 
K. Tsigklifis and A.D. Lucey, Modelling and analysis of the global stability of Blasius boundary-layer

At the wall the boundary conditions $u_{x}(x, 0, t)=u_{z}(x, 0, t)=0$ are enforced for the rigid-wall portions $x_{s} \leq x<x c_{s}$ and $x c_{o}<x \leq x_{o}$. On the compliant-wall section the velocity and stress components are continuous between fluid and solid. Thus, the linearized boundary conditions for the velocity are

$u_{x}(x, 0, t)+\eta(x, t) \partial U_{x} / \partial z(x, 0, t)=0, \quad u_{z}(x, 0, t)=\partial \eta / \partial t(x, t), \quad x c_{s} \leq x \leq x c_{o}$

The pressure perturbation at the wall is obtained by integrating the linearized $z$-momentum equation of the Navier-Stokes equations between the wall and infinity, taking into account that disturbance pressure must vanish at infinity, i.e.

$$
p(x, 0, t)=\int_{0}^{L_{H}}\left(\frac{\partial u_{z}}{\partial t}+\frac{\partial U_{z}}{\partial x} u_{x}+\frac{\partial U_{z}}{\partial z} u_{z}+U_{x} \frac{\partial u_{z}}{\partial x}+U_{z} \frac{\partial u_{z}}{\partial z}+\frac{1}{\gamma \sqrt{x \mathrm{Re}_{s}}} \frac{\partial \omega_{y}}{\partial x}\right) \gamma \sqrt{x / \mathrm{Re}_{s}} d H
$$

where, $L_{H}$ is the total height of domain, made large enough to ensure that disturbances of the vorticity and the $z$ component of the velocity vanish infinitely far from the wall, giving

$\omega_{y}\left(x, L_{H}, t\right)=0, \quad u_{z}\left(x, L_{H}, t\right)=0$

Eigenvalue form of the problem

We proceed by applying the decompositions,

$\omega_{y}(x, z, t)=\hat{\omega}(x, z) e^{\lambda t}, \quad \sigma(x, z, t)=\hat{\sigma}(x, z) e^{\lambda t}, u_{x}(x, z, t)=\hat{u}_{x}(x, z) e^{\lambda t}, \quad u_{z}(x, z, t)=\hat{u}_{z}(x, z) e^{\lambda t}$, $\eta(x, t)=\hat{\eta}(x) e^{\lambda t}, \quad p(x, 0, t)=\hat{p}(x, 0) e^{\lambda t}$

with $\lambda=-i \omega$, to the linear system of Eqns. (2), (4) and (5), taking into account the boundary conditions (7), $(8),(9)$ and (10) to obtain a generalized eigenvalue system,

$\left[C_{2}\right]\{\hat{X}\}=\lambda\left[C_{1}\right]\{\hat{X}\}, \quad\{\hat{X}\}=\left\{\begin{array}{llll}\hat{\omega} & \hat{\sigma} & \hat{\eta} & \hat{\varphi}\end{array}\right\}^{T}$,

with $\hat{\varphi}=\lambda \hat{\eta}$, from which the eigenvalues $\lambda$ and eigenvectors $\{\hat{X}\}$ can be extracted. If the real part of an eigenvalue $\lambda$ is positive, instability in time is to be expected, otherwise disturbances will decay.

Non modal stability analysis

The decompositions of Eqn. (11) are valid as $t \rightarrow \infty$ and so the eigenvalues and the corresponding eigenvectors represent only the temporal asymptotic behavior. In order to investigate the transient behavior of the FSI system (e.g. Schmid, 2007) we define the energy norm of the system to be

$$
E(t)=1 / 2 \int_{0}^{L_{H}} \int_{x_{s}}^{x_{o}}\left(\left|u_{x}\right|^{2}+\left|u_{z}\right|^{2}\right) d x d H+1 / 2 \int_{x_{c s}}^{x_{c o}}\left(I C \dot{\eta}^{2}+B C\left(\frac{\partial^{2} \eta}{\partial x^{2}}\right)^{2}+K C \eta^{2}\right) d x
$$

where the flow kinetic energy comprises the first integral while the kinetic and potential (plate strain plus spring) energy of the structure constitute the second integral; we look for initial disturbances which maximize the energy at time $t$,

$$
G(t)=\max _{\vec{X}_{0} \neq 0} \operatorname{ax}_{\|\vec{X}(t)\|_{E}^{2}} .
$$

The disturbances are constructed by the linear superposition of the two-dimensional temporal modes,

$$
\vec{X}(x, H, t)=\sum_{j=1}^{N} k_{j}(t) \hat{\vec{X}}_{j}(x, H) \cdot
$$

Substituting the above expression into the initial-value form of the problem and taking into account that eigenmodes satisfy the generalized eigenvalue problem, (12), the maximum energy growth finally takes the form $G(t)=\left\|\underline{\underline{F}} \exp (\underline{\underline{\Lambda}} t) \underline{\underline{F}}^{-1}\right\|_{2}^{2}$, with $\Lambda_{j j}=\lambda_{j}=-i \omega_{j}$ and $M=F^{T} F \quad$ is the Cholesky decomposition of the Gramian matrix M with entries, 
K. Tsigklifis and A.D. Lucey, Modelling and analysis of the global stability of Blasius boundary-layer

$$
M_{i j}=1 / 2 \int_{x_{s}}^{x_{o}} \int_{0}^{L_{H}}\left(u_{x, i}^{*} u_{x, j}+u_{z, i}^{*} u_{z, j}\right) d H d x+1 / 2 \int_{x_{c s}}^{x_{c o}}\left(I C \dot{\eta}_{i}^{*} \dot{\eta}_{j}+\left.\left.B C \frac{\partial^{2} \eta^{*}}{\partial x^{2}}\right|_{i} \frac{\partial^{2} \eta}{\partial x^{2}}\right|_{j}+K C \eta_{i}^{*} \eta_{j}\right) d x
$$

where $*$ denotes the complex conjugate. The largest growth at time $t$ is given by the largest singular value of $\underline{\underline{F}} \exp (\underline{\underline{\Lambda}}) \underline{\underline{F}}^{-1}$ and the initial condition that provides it is given by $\vec{k}_{0}=\underline{\underline{F}}^{-1} \vec{z}$, with $\vec{z}$ the right singular vector.

\section{NUMERICAL SOLUTION}

\subsection{Numerical approximation}

A second-order finite-difference method is used for discretization in $x$ direction and a Chebyshev pseudospectral method is exploited for the normal direction. The flow domain is divided in $M=M_{w 1}+M_{c}+M_{w 2}$ intervals in the streamwise direction, where $M_{w 1}, M_{c}$, and $M_{w 2}$ are respectively the number of fluid cells over the rigid-wall 1, the compliant-wall and the rigid-wall 2 sections, while $\mathrm{N}+1$ points are deployed in the normal direction and the linear transformation used to map the collocation points from the interval $[1,0]$ to $\left[0, L_{H}\right]$ is

$$
H_{k}=L_{H}(1-\cos (k \pi / 2 N)), k=0, N \text {. }
$$

Then, the discretized form of the momentum Eqn. (2) is written for each cell $(i, j)$ :

$$
\begin{aligned}
& \left.\left.\frac{\partial \Omega_{y}}{\partial x}\right|_{i j} \hat{u}_{x}\right|_{i j}+\left.\left.\frac{\partial \Omega_{y}}{\partial z}\right|_{i j} \hat{u}_{z}\right|_{i j}+\left.U_{x}\right|_{i j} \frac{3 \hat{\omega}_{i j}-4 \hat{\omega}_{i(j-1)}+\hat{\omega}_{i(j-2)}}{2 \Delta x}+\left.U_{z}\right|_{i j} \sum_{k=1}^{N} D_{i j, k j} \hat{\omega}_{k j}+ \\
& -\frac{1}{\gamma \sqrt{x_{j} \operatorname{Re}_{s}}} \frac{\hat{\omega}_{i(j+1)}-2 \hat{\omega}_{i j}+\hat{\omega}_{i(j-1)}}{\Delta x^{2}}-\frac{1}{\gamma \sqrt{x_{j} \operatorname{Re}_{s}}} \sum_{k=1}^{N} D_{i j, k j}^{2} \hat{\omega}_{k j}=-\lambda \hat{\omega}_{i j}, \quad i=2, N-1 \quad \& \quad j=2, M-1
\end{aligned}
$$

where $[D]$ and $[D]^{2}$ are respectively the Chebyshev differentiation matrices for the first and second derivative at the collocation points. The Helmholtz decomposition, Eqn. (4), is approximated by zero-order vortex sheets and zero-order source sheets at the fluid-solid interface. Finally, the Arpack library has been implemented in order to extract a significant part of the spectrum of Eqn. (12), namely 1500 eigenvalues and their respective eigenvectors, using a relatively large Krylov subspace of 4500 vectors.

\subsection{Choice of system parameters and validation of the numerical scheme}

The wall parameters were selected in such a way that the numerically calculated critical velocity for the onset of the divergence instability in potential flow over a finite compliant wall (Pitman \& Lucey, 2009) was well above the free stream velocity $U_{\infty}^{\prime}=10 \mathrm{~m} / \mathrm{s}$ used herein. In order to validate the numerical methodology for the global stability outlined above we first perform a mesh independence test. In Fig. 2a the eigenvalue spectrum in the $\omega$-plane is shown for three different mesh sizes, $150 \times 55,180 \times 65$ and $240 \times 75$. There is convergence to a solution, mainly for the eigenvalues around the real axis; moving away from the real axis the convergence deteriorates. This poses a limitation to the number of modes that can be used for the disturbance expansion, Eqn. (15), regarding the transient FSI system behavior. Nevertheless, the convergent discrete modes that contribute to the transient as well as to the temporal asymptotic behavior could be evaluated. A further test can be implemented through comparison with the local stability analysis. More specifically, following Ehrenstein and Gallaire (2005), the spatial structure of the two-dimensional global temporal modes exhibits a growth in amplitude when progressing downstream, which may be quantified by computing

$$
\frac{A(x)}{A\left(x_{s}\right)}=\frac{\sqrt{\int_{0}^{L_{H}}\left(\hat{u}_{x}^{*}(x, H) \hat{u}_{x}(x, H)+\hat{u}_{z}^{*}(x, H) \hat{u}_{z}(x, H)\right) d H}}{\sqrt{\int_{0}^{L_{H}}\left(\hat{u}_{x}^{*}\left(x_{s}, H\right) \hat{u}_{x}\left(x_{s}, H\right)+\hat{u}_{z}^{*}\left(x_{s}, H\right) \hat{u}_{z}\left(x_{s}, H\right)\right) d H}}
$$

This spatial growth may be compared to the amplitude growth due to convective instability as predicted by the local parallel-flow assumption. For each streamwise location $x$, the spatial Orr-Sommerfeld equation is solved for the complex wavenumber $a_{x}$ for known local complex frequency $\omega_{o}=\left(\mathrm{Re}_{o} / \mathrm{Re}_{s}\right) \omega_{s}$. Then, the 
amplitude growth as predicted by the local analysis for each streamwise location is given by $A(x) / A\left(x_{s}\right)=\exp \left(\int_{x_{s}}^{x}-a_{i, x} d x\right)$ with $a_{i, x}$, the imaginary part of the wavenumber $a_{x}$. A comparison between the streamwise global mode growth and that predicted by the local stability analysis has been performed at the frequency of the temporally least stable Tollmien-Schlichting wave (TSW), denoted by the blue arrow in Fig. $2 \mathrm{a}$. There is good agreement in the slope of the lines in Fig. 2b, noting that the wall leading edge exerts a small upstream influence, while the trailing edge a stronger downstream influence due to the parabolic nature of the flow.
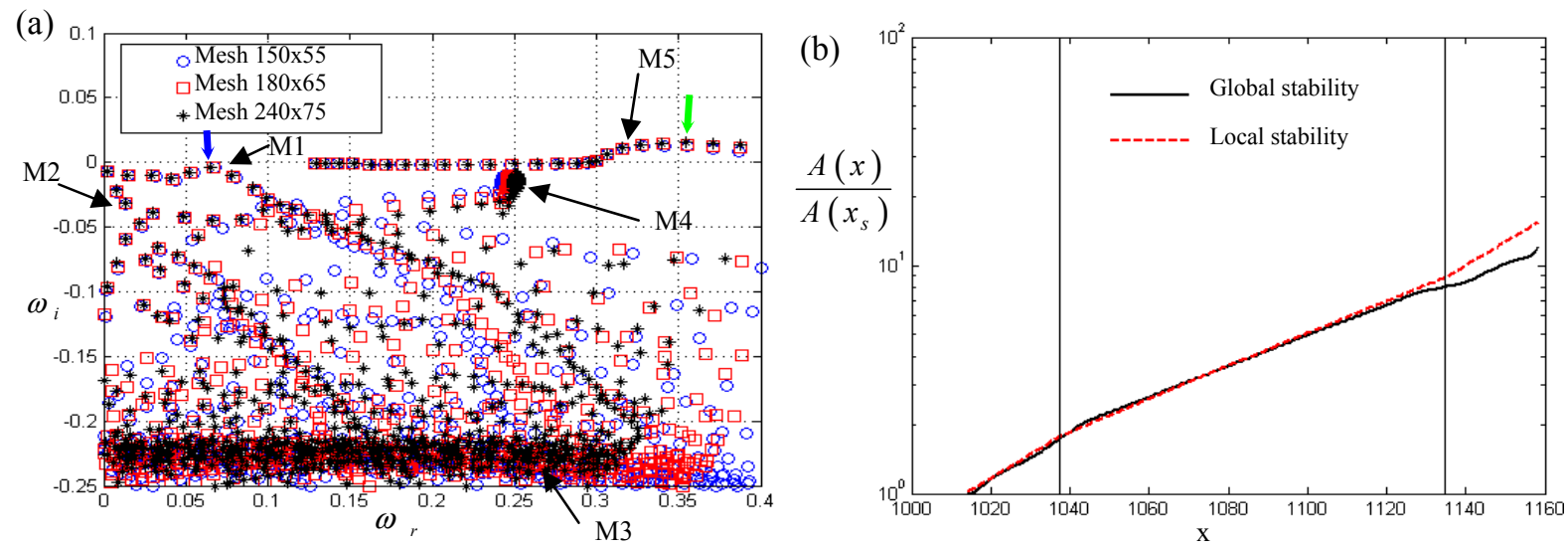

Figure 2. (a) The $\omega$-plane eigenvalue-spectrum predicted by the global stability for different mesh sizes. (b) Spatial amplification rates of the most unstable in time TS mode denoted by blue arrow in (a); the vertical solid lines signify the start and the end of the compliant wall.. Flow and compliant-wall parameters:

$$
\begin{aligned}
& \operatorname{Re}_{s}=3000, U_{\infty}^{\prime}=10 \mathrm{~m} / \mathrm{s}, \rho_{l}^{\prime}=1000 \mathrm{~kg} / \mathrm{m}^{3}, \mu_{l}^{\prime}=1.37 \times 10^{-3} \mathrm{Pas}, \omega_{s}=0.07755, L_{w 1}^{\prime}=0.01 \mathrm{~m}, L_{c}^{\prime}=0.04 \mathrm{~m}, \\
& L_{w 2}^{\prime}=0.01 \mathrm{~m}, \rho_{m}^{\prime}=1000 \mathrm{~kg} / \mathrm{m}^{3}, h_{m}^{\prime}=2 \times 10^{-3} \mathrm{~m}, D^{\prime}=0 \mathrm{~kg} / \mathrm{m}^{2} \mathrm{~s}, B^{\prime}=8.89 \times 10^{-6} \mathrm{Nm}, K^{\prime}=115 \times 10^{6} \mathrm{~N} / \mathrm{m}^{3}
\end{aligned}
$$

\section{RESULTS AND DISCUSSION}

The eigen-spectrum in Fig. 2a reveals a variety of (labelled) instability branches. As identified by Åckerick et al. (2008), there are three types of modes associated with the boundary layer flow over a rigid wall: the branch associated with the TSWs labelled M1, the Orr mode branch labelled M2, and the modes associated with the wall normal continuous branch labelled M3. The M4 branch is also related to the continuous spectrum of the local Orr-Sommerfeld analysis of the Blasius boundary layer flow due to the inlet boundary condition for the normal velocity. As the compliant wall is also a wave-bearing medium, an additional branch associated with its structural modes contributes to the total eigen-spectrum and is labelled M5. These modes are neutrally stable in time or slightly damped and, depending on their position in the $\omega$-plane, can extract energy through resonance with either fluid-based TSWs or the wall-based travelling-wave flutter (TWF) convective instabilities, giving rise to a global instability in time. The $\omega$-plane position of the M5 branch depends upon the following in vacuo structural eigen-frequencies modified by the fluid load,

$$
\omega_{n, \text { analyt. }}=\sqrt{-D C^{2}+4 I C \cdot B C\left(\frac{n \pi}{L_{c}}\right)^{4}+4 I C \cdot K C} / 2 I C
$$

The modes for which instability in time is predicted are labelled by the green arrow in Fig. 2a. These are realised in the local $\alpha$-plane as a combination of a spatially amplified divergence mode (D) with a spatially unstable TWF mode; these are shown in Fig. 3a. The power spectrum of the wall displacement, Fig. 3b, shows good agreement with the local analysis for the wavelength of the divergence mode but some difference for the wavelength of the TWF mode. This difference is attributed to the coexistence of the two modes on the compliant wall interface; as we move away from the wall, the wavelength of the velocity disturbances tends to that predicted by the local stability for the TWF mode, while the divergence mode vanishes.

To illustrate transient behaviour of the FSI system, Fig. 4 shows that a compliant wall free from resonancetype instability advects lower maximum energy downstream than a rigid wall, but of the same order of magnitude. In this case the inclusion of structural damping has a marginally destabilizing effect on the transient response of the compliant wall. However, corresponding results (nor presented here) for which resonance occurs show that damping leads to significantly higher (up to three times) levels of transient growth and advection. 
K. Tsigklifis and A.D. Lucey, Modelling and analysis of the global stability of Blasius boundary-layer
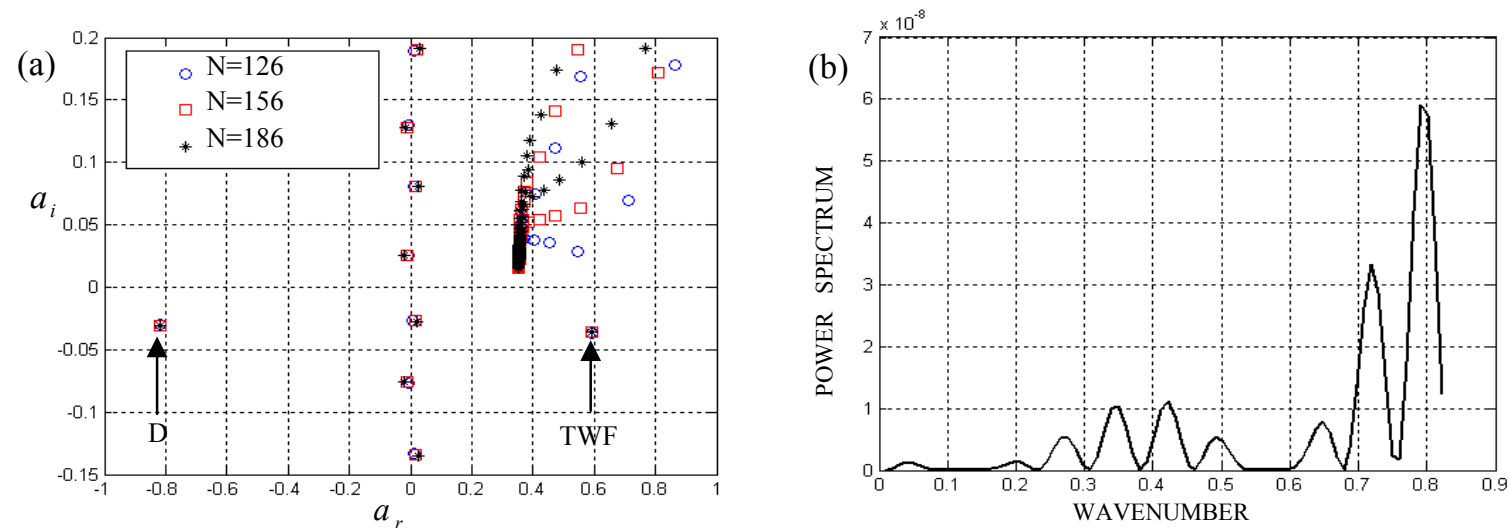

Figure 3. Local and global representations of the most temporally unstable TWF mode, denoted by the green arrow in Fig. 2a. (a) $\alpha$-plane eigenvalue spectrum predicted by local stability analysis in the middle of the domain $\left(\mathrm{Re}_{\mathrm{x}}=3105\right)$ for different numbers of Chebyshev-Gauss-Lobatto points, (b) power spectrum of the wall displacement versus the wavenumber. Flow and compliant wall parameters as those in Fig. 2

Figure 4. Transient response: maximum growth of the energy $G(t)$, as a function of time t. Flow and compliant wall parameters:

$$
\begin{aligned}
\operatorname{Re}_{s} & =1000, U_{\infty}^{\prime}=10 \mathrm{~m} / \mathrm{s}, \rho_{l}^{\prime}=1000 \mathrm{~kg} / \mathrm{m}^{3}, \mu_{l}^{\prime}=1.37 \times 10^{-3} \mathrm{Pas}, \\
\omega_{s} & =0.07755, L_{w 1}^{\prime}=0.01 \mathrm{~m}, L_{c}^{\prime}=0.01 \mathrm{~m}, L_{w 2}^{\prime}=0.01 \mathrm{~m}, \\
\rho_{m}^{\prime} & =1000 \mathrm{~kg} / \mathrm{m}^{3}, h_{m}^{\prime}=2 \times 10^{-3} \mathrm{~m}, B^{\prime}=8.89 \times 10^{-5} \mathrm{Nm}, K^{\prime}=400 \times 10^{6} \mathrm{~N} / \mathrm{m}^{3}
\end{aligned}
$$

\section{ACKNOWLEDGMENT}

The authors wish to acknowledge the financial support of the Australian Research Council (ARC) through the Discovery Projects scheme (Grant DP1096376).

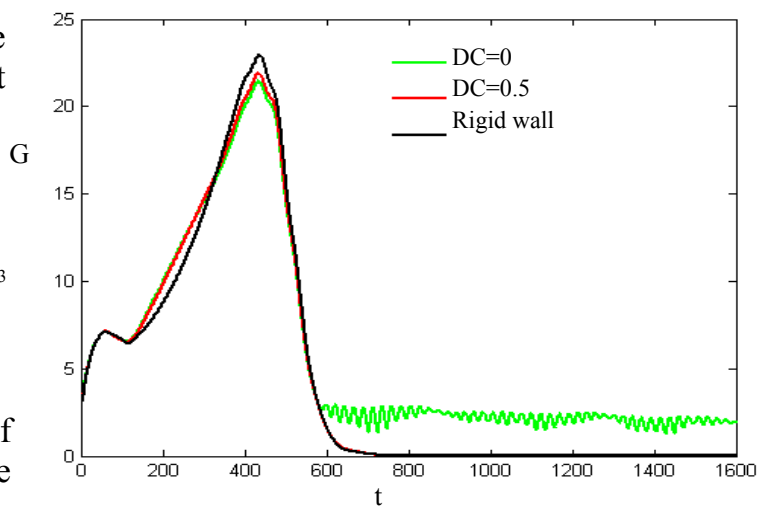

\section{REFERENCES}

Åckerick, E., Ehrenstein, U., Gallaire, F., Henningson, D.S. (2008). Global two-dimensional stability measures of the flat plate boundary-layer flow. European Journal of Mechanics B/Fluids, 27: 501-513.

Carpenter, P.W. and Garrad, A.D. (1985). The hydrodynamic stability of flows over Kramer-type compliant surfaces: Part 1. Tollmien-Schlichting instabilities. Journal of Fluid Mechanics, 155:465-510.

Davies, C. and Carpenter, P.W. (2001). A novel velocity-vorticity formulation of the Navier-Stokes equations with applications to boundary layer disturbance evolution. Journal of Computational Physics, 172:119-165.

Dixon, A.E., Lucey, A.D. and Carpenter, P.W. (1994) The optimization of viscoelastic walls for transition delay. AIAA Journal, 32:256-267.

Ehrenstein, U. and Gallaire, F. (2005). On two-dimensional temporal modes in spatially evolving open flows: the flat-plate boundary layer. Journal of Fluid Mechanics, 536:209-218.

Kempka, S.N., Strickland J.H., Glass M.W., Peery J.S., Ingber M.S.(1995).Velocity boundary conditions for vorticity formulations of the incompressible Navier-Stokes equations. Forum on vortex methods for engineering applications, sponsored by Sandia National Labs, pp.1-22, Albuquerque, NM

Lucey, A.D., and Carpenter, P.W. (1992). A numerical simulation of the interaction of a compliant wall and inviscid flow. Journal of Fluid Mechanics, 234:121-146.

Pitman, M. W. and Lucey, A. D. (2009). On the direct determination of the eigenmodes of finite flowstructure systems, Proceedings of the Royal Society A, 465:257-281.

Pitman, M.W. and Lucey, A.D. (2010). Stability of plane-Poiseuille flow interacting with a finite compliant panel. In: Proc. of the 17th Australasian Fluid Mechanics conference, Auckland, New Zealand, pp.563566.

Schmid, P.J.(2007). Nonmodal stability theory. Annual Review of Fluid Mechanics, 39:129-162. 